\title{
The SWOT Analysis of the Intangible Cultural Heritage of Changle Story
}

\author{
Wei Tu and Jie Zheng \\ School of Art and Design of Huang Jia Hu Campus, \\ Wuhan University of Science and Technology, Qingling Street, Hongshan District, \\ Wuhan, Hubei Province \\ South 10, Huang Jia Hu Campus, Wuhan University of Science and Technology, \\ Qingling Street, Hongshan District, Wuhan, Hubei Province \\ 381686581@qq.com,847653277@qq.com
}

Keywords: Changle story; Intangible cultural heritage; SWOT Analysis

\begin{abstract}
Changle story, which has been listed as a state-level intangible cultural heritage, is a priceless cultural treasure to the whole world. It is quite necessary and worthwhile for us to research and explore how to make the fullest use of its cultural and economic values. The current paper will carry out the method of SWOT analysis to do some research on the advantages, disadvantages, opportunities and threats of inheriting and preserving Changle Story, aimed at figuring out some effective strategies in better inheriting and preserving it so that it will always exist under the survival rule of modern society.
\end{abstract}

\section{Introduction}

Following the trend of globalization and modernization, we must be soberly aware of the survival status of our intangible cultural heritages. Changle Story contains rich folk customs and cultural background, in which a lot of folk stories are remade and played in real-world scenes with the only use of simple body language. As the most representative intangible cultural heritage of Changle Ancient Town, Changle Story is rich in content and characterized by its distinctive local style, making it very popular among the public. However, it is a burning problem to be solved how we can continuously and effectively inherit and preserve Changle Story in the process of urbanization.

\section{Changle Story}

Originated from Sui and Tang Dynasty, Changle Story flourished in Ming and Qing Dynasty, and peaked in the 1980s. It is not like telling ordinary stories, but an ancient and mysterious folk art in which various kinds of stories are played by people dressed up in a traditional way with the use of human body language. The stories or plots used for the "dressing-up" are adapted from legendary tales, historical romance, literature samples, and current affairs that have spread around Changle Ancient Town of Miluo since the State of Chu (a nation during the Spring and Autumn Period). Combinining the fine arts, acting, history, literature, astronomy, geography, folk customs and the spirit of the times, Changle Story is divided into four categories - ground stories, ground and stage stories, Gaocai stories and stilting stories. It is developed from yearly sacrificial practices during lunar January and the lantern show during Shangyuan Period (a time in Song Dynasty). On each of the first half month of lunar Juanuary, residents in Changle will spontaneously divide themselves into two groups - group of Shangshi Street and group of Xiashi Street, and all the people - the old and the young, men and women - will dress up themselves in colorful clothes and play various kinds of wonderful folk stories using their bodies and souls. Though lasting as long as half a month, it is always filled with joy and excitement, and people there never slack off in their acting. The whole town is really bustling and exciting, with local villagers being performers, and alleys, halls and fields of the town used as their stage[1], and local living dramas and comedies played during the half month. 
Changle Story is a country art shared and jointly involved by the residents of the town, in which a lot of folk stories are remade and played in real-world scenes with the only use of simple body language. As an art born in local country life, it reveals human's instinctive creativity and aesthetic ability. Changle Story is like a bridge connecting the reality and dream, and preserves the instinctive attributes of initial primitive art - to balance the spirit and soul among human. With the power of pure beauty, it quietly survives and exists among the villagers at the bottom of the society in the form of innovative and characteristic art.

\section{Introduction}

Following the trend of globalization and modernization, we must be soberly aware of the survival status of our intangible cultural heritages. Changle Story contains rich folk customs and cultural background, in which a lot of folk stories are remade and played in real-world scenes with the only use of simple body language. As the most representative intangible cultural heritage of Changle Ancient Town, Changle Story is rich in content and characterized by its distinctive local style, making it very popular among the public. However, it is a burning problem to be solved how we can continuously and effectively inherit and preserve Changle Story in the process of urbanization.

\section{Changle Story}

Originated from Sui and Tang Dynasty, Changle Story flourished in Ming and Qing Dynasty, and peaked in the 1980s. It is not like telling ordinary stories, but an ancient and mysterious folk art in which various kinds of stories are played by people dressed up in a traditional way with the use of human body language. The stories or plots used for the "dressing-up" are adapted from legendary tales, historical romance, literature samples, and current affairs that have spread around Changle Ancient Town of Miluo since the State of Chu (a nation during the Spring and Autumn Period). Combining the fine arts, acting, history, literature, astronomy, geography, folk customs and the spirit of the times, Changle Story is divided into four categories - ground stories, ground and stage stories, Garcia stories and stilting stories. It is developed from yearly sacrificial practices during lunar January and the lantern show during Shang yuan Period (a time in Song Dynasty). On each of the first half month of lunar January, residents in Changle will spontaneously divide themselves into two groups - group of Shangshi Street and group of Xiashi Street, and all the people - the old and the young, men and women - will dress up themselves in colorful clothes and play various kinds of wonderful folk stories using their bodies and souls. Though lasting as long as half a month, it is always filled with joy and excitement, and people there never slack off in their acting. The whole town is really bustling and exciting, with local villagers being performers, and alleys, halls and fields of the town used as their stage[1], and local living dramas and comedies played during the half month.

Changle Story is a country art shared and jointly involved by the residents of the town, in which a lot of folk stories are remade and played in real-world scenes with the only use of simple body language. As an art born in local country life, it reveals human's instinctive creativity and aesthetic ability. Changle Story is like a bridge connecting the reality and dream, and preserves the instinctive attributes of initial primitive art - to balance the spirit and soul among human. With the power of pure beauty, it quietly survives and exists among the villagers at the bottom of the society in the form of innovative and characteristic art.

\section{The SWOT Analyses of Changle Story's Intangible Cultural Heritage}

The SWOT analysis system is an effective method which helps researchers objectively and accurately study and analyses the current situation of a certain organization. In other words, it is a tool for the selection of proper strategies combining internal factors and external factors in the four dimensions of strengths, weaknesses, opportunities and threats - the internal factors contain the 
dimensions of strengths and weaknesses, and the external factors contain the dimensions of opportunities and threats[2]. Finally, based on the SWOT analysis, the researchers will figure out proper solutions or strategic tactics to the existing problems.

The Analysis of Strengths(S). The analysis of strength will focus on what are favorable factors of Changle Story as a state-level intangible cultural heritage, which give it some advantages over other intangible cultural heritages, for those favorable factors are historically inherited, making Changle Story impossible to clone but easy to develop. To have a better understanding of the development of Changle Story, a priceless cultural treasure, the author has interviewed the inheritor of it, through which we can conclude the strengths of this intangible cultural heritage as follows:

The Rich Cultural Deposits of Changle Story Contributes to its Inheriting. Regarded as the memories of the existing culture, intangible cultural heritages play an essential role in human community. As is known to everyone, man can not just live in the moment. That is to say, man can not live without the memories of history, especially the collective memories of social and cultural life. Changle, a millennium town with a strong cultural atmosphere, is nestled between hills and rivers, which fosters its natural aura; moreover, it is saturated with the spirit born in the grand southern land of ancient China - "The way stretches endless ahead, I shall search heaven and earth"(By Qu Yuan, a great poet and politician in ancient Chu). Influenced by the beautiful landscape and ancient Chu culture, inherited and developed by generations of local residents, Changle Story forms a unique regional feature and shows the charm of Chu culture. It is the profound and well-preserved culture that makes Changle Story lively and longstanding. History is not only the gone and static past but living things in being, which is like a channel connecting the past, the present and the future. What makes Changle Story remain its rich cultural deposits and inheriting of generations of local residents. For this reason, intangible cultural heritages are called living cultures. Any living culture can not be fostered and developed without a strong cultural atmosphere that contributes to the inheriting of Changle Story.

It is Uniquely Characteristic and Impossible to be Cloned. Rooted in the rural life of Changle Town, Changle Story is a simple and free imagery art, which is created by the local villagers in their laboring and social life to meet their need of spirit and soul. In the eyes of outsiders, all the residents of Changle are united as a whole; in fact, they are divided into two rivalry groups - group of Shangshi Street and group of Xiashi Street. The core of Changle Story is competition, including the competition in content, form and reactivity, which goes through the period of Changle Story from lunar January 1st to 15th. This unique regional feature gives Changle Story an advantage over other intangible cultural heritages and makes it impossible to be cloned. Just like a skillful and talented worker can not be replaced by other workers, Changle Story intangible cultural heritage is very competitive in inheriting and preservation.

It is Publicly Involved and has Great Potential for Exploitation. Most intangible cultural heritages come from civil society, and thus the most suitable preserving approach and environment for them also exist in civil society. For a long time, the preservation of intangible cultural heritages in China are undertaken by the government, but the public have a weak sense of being involved in it. Therefore, it is necessary and urgent to get all the people involved in the preservation of intangible cultural heritages. Actually, the public are not very concerned about whether the region they live in has high market development potential. However, all-people involvement provides the public with access to the current situation of intangible cultural heritages and meanwhile creates a lively atmosphere for the preservation of intangible cultural heritages, which is reflected in Changle Story. All the villagers in the ancient town spontaneously gather around to celebrate the big festival, which gradually becomes a common habit among the local people. Each family and everyone, old as a hundred years old and young as only one year old, could be the participators and players of the stories, and they spontaneously get involved in them heart and soul. As a popular activity supported and favored by a large number of people, Changle Story has a stable social environment and thus has great potential for market development. In a word, Changle Story is an intangible cultural heritage with public support and involvement, and its potential for market development can be never underestimated. 
The Analysis of Weaknesses(W). Although Changle Story intangible cultural heritage has an advantage in the inheriting and preservation over others, it will be restricted by its weaknesses. Therefore, there is a strategic point in analyzing its disadvantageous factors.

Limited Space. The ancient Changle street is oriented south and north. Borderd by the Pizhao pavilion, the street is divided in to Xiashi street in the south and Shangshi street in the north. The granites of the ancient street are where Changle Story was born. With the development of Changle Story, the ancient street filled with granites now is not large enough to hold an increasing number of full-scale shows. Although the authority of the town has transferred the arena from the ancient street to the center street paved by cement, the center street is also crowded with oceans of people during the Spring festival. The limited space of the town provides few options for the arena of the acting shows, which has become a block of inheriting and preserving Changle Story.

Lack of Investors. The industrialized development of intangible cultural heritages requires to be led by some investors, without whose help and support their industrialized development will be baseless, just like a water without source. In terms of Changle Story, it is an unparalleled activity whose traditions are passed on from generation to generation for thousands of years, and an incomparable art valued by all the people in the town. Each family in the town, rich or poor, regardless of classes, voluntarily help collect funds for the activity, never demanding any rewards. Changle Story began its industrialized development just a few years ago, but the strength of local people is too limited to support its industrialized development on a large scale. What's more, they are not professional investors and have limited financial resources, and for this reason, they can never be the leading and key investors of the industrialized development of Changle Story. As a result, the industrialized development of Changle Story is badly blocked by the lack of investors.

The Analysis of Opportunities $(\mathbf{O})$. The analysis of opportunities and threats refer to the external factors that affect the development of a certain subject. Uncontrollable as those factors are, they are functioned as references to help make strategic decisions. We can take advantage of the opportunities but avoid the threats as much as possible. The paper will conclude the opportunities that probably contribute to the inheriting and preservation of Changle Story.

The Increased Governmental and Public Awareness of Preserving Changle Story Intangible Cultural Heritage. Recently, a boom of preserving intangible cultural heritages has emerged among the public, and the government will attach greater importance to Changle Story, the state-level intangible cultural heritage. Miluo government is now seizing every favorable opportunity to do a solid job to preserve Changle Story intangible cultural heritage. Firstly, Miluo government summoned the authorities of Changle in Miluo, Zhangyi in Chenzhou, Zhumei in Lianyuan, Majitang in Yiyang, and Huangyangsi in Yongzhou to co-sign the Cooperation Agreement of Hunan Taige Story Alliance, agreeing on more intensified cooperation in inheriting and preserving Changle Story in terms of developing goals, regional communication and industry convergence. Secondly, the first Taige Story Festival will be held by Miluo government to promote the popularity of Changle Story. Thirdly, the government will spare no effort to build the Story Town and an industrial park featuring Changle stories, making Changle a unique and characteristic town combining culture, tourism and stories. What Miluo government has done will lay the root for the creation and production of stories, scale operation and industrialized development of Changle Story. In addition, the decision-makers of Changle town have put forward a slogan - Popularize the business card of Changle Story as a state-level intangible cultural heritage, build the unique and characteristic Story Town, which promotes the popularity and publicity of Changle Story reach a higher spot. Saturated with historical spirit and burdened with cultural inheriting, Changle Story gives life to the town of stories and makes it longstanding. Now preserving Changle Story intangible heritage has become the cultural self-consciousness of 30,000 residents in the town.

The Diversity of Teaching Mode. Fan Xing, an old man who is the inheritor of Changle Story, has applied for making it a special course of local schools with the aim of better inheriting the unique folk art of Changle town. In the primary schools of Changle, specially-designed acitivities of Changle Story are open to students in PE lessons, for example, stilting stories, and some professional teachers are invited to instruct the students in the rehearsal. Students of the town have 
been influenced by the local folk art from childhood, and thus it has been deeply rooted in them. What's more, a series of textbooks are being written, including the sections of the origin, values, stories, performers, inheriting and spreading of Changle Story, and those textbooks are planned to be applied in local schools. The diverse teaching modes of Changle Story will help the inheriting and preservation of unique folk art take firm root among local people.

The Alliance of Cultural Resources. On February 10th, 2017, the conference of Hunan Taige Story Alliance was successfully held in Changle town, Miluo. Hunan Taige Story Alliance is made up of state-level Changle Story of Miluo, state-level Yizhang Night Story of Chenzhou, state-level Zhumei Tai Story of Lianyuan, province-level Majitang Zha Story of Tao Jiang in Yiyang City, and province-level Huangyangsi Zha Story of Yongzhou. The alliance of Taige Story from five towns will not only be conductive to the continuation of traditional customs of the town, but also contribute to the inheriting, preservation and development of the culture of Hunan Taige Story. More importantly, it will help Changle Story - the state-level intangible cultural heritage-deeply rooted in local people's life and serve the local community. The alliance of Taige Story can be reinforced by the following ways: regularly hold Taige Story Festivals, make regional communication and exchange visits, establish the personnel training and cooperating system of Taige Story, occasionally hold intangible cultural heritage academic conferences. In a word, the alliance of cultural resources from the five towns will play a key role in the preservation of the intangible cultural heritage.

The Analysis of Threats(T). Threats from modern civilization and commercialization. There is no denying that it is the countryside sensibility and affinity hidden behind Changle Story that give life to it and make it longstanding. However, this state-level intangible cultural heritage is being faced with threats from modern civilization that more and more local people follow the trend of the time to make a living in the city, keeping away from their tradition. As we can see, the environment where the traditional culture has changed a lot and local people are easily influenced by modern social values, which make it a challenging job to inherit and preserve Changle Story. Changle Story titled a state-level intangible cultural heritage has won fame and economic benefits for the town and local people. Therefore, in order to ensure the steady and sustainable development of Changle Story, we have to cut down some utilitarian commercial exploitation and excavate the cultural content deep inside it, and thus it will emit a fascinating brilliance of traditional folk art and lay the root in the public.

The Fragile Inheriting System. For the moment, intangible cultural heritages, a term born in contemporary in heritage movement, are based on local cultures, and naturally local people are the inheritors of them[3]. As for Changle Story, the inheriting of it is more than copying the traditional culture but updating and rebuilding it, which is a heavy burden we are supposed to shoulder. As everyone knows, much of the inheriting and preservation of traditional cultures are undertaken by the elderly inheritors, but the conservative traditional values deeply rooted in their mind make it difficult for them to keep up with the rapid development of the Information Period, and gradually they are far from innovation. As for the young people of the town, they are not willing to give up their city life and stay in the town to preserve the traditional culture. Influenced by the factors above, the inheriting system of Changle Story is very fragile and for this reason, the development of the rational culture will be inevitably affected.

Lack of Market Competition. Currently, originally imitating and performing are the basic ways to inherit and preserve Changle Story, but its outmoded language and simplex plot can't meet the needs of modern society. Compared with other cultural brands, Changle Story has no advantage in the market competition. In terms of development, a cultural brand is formed and created just because it shows and meets human's emotional needs, or that's to say, it reflects the lifestyle and living attitude of modern people. Therefore, we can never ignore the value of brand. When it comes to Changle Story, its cultural brand has not been formed because local people have a weak sense of brand and are not aware of the significance of exploiting its cultural and economic values, which will undoubtedly leads to the weak market competitiveness of Changle story. 


\section{Some Strategies in Better Inheriting and Preserving Changle Story Intangible Cultural Heritage}

Strengthen the Publicity and Promotion of Changle Story and Build Its Own Cultural Brand. Currently, Changle Story is only known to people in some towns of Hunan, and not so many people know and pay close attention to it nationwide. The following ways can be effective to strengthen the publicity and promotion of Changle Story and build its own cultural brand: Firstly, greater efforts must be made to promote its publicity and make it popular among more people. In particular, we must take advantage of the golden period of the Spring Festival to publicize Changle Story through various traditional media, and to arouse the public affection to this traditional folk culture and customs. Secondly, activities such as the Story Festival must be regularly held in Changle town, Miluo City, Hunan province and nationwide. We should market Changle Story deeply and build the cultural brand of Changle town. Thirdly, a specialist website must be established to exhibit the inheritors and representative works of Changle Story. Fourthly, we can also draw the attention of more potential guests and investors to Changle Story by joining in Folk Arts Festivals of different places. And in this way we can develop a larger market for Changle Story. Lastly, we can create live stories of various genres and types adapted from legendary tales, historical romance, literature samples, and current affairs that are included in Changle Story. For example, we can create cartoons, TV series, shadow plays and movies based on Changle Story to expand its popularity and influence among the public, and finally build its own cultural brand.

Strengthen the innovation of Changle Story. Innovation is not only the soul of Changle Story, but also an inexhaustible motive force for it to develop and the source for it to keep vigor. Along with the rapid development of modern society, it is of necessity to innovate in Changle Story from multiple perspectives. First of all, it is a must to strengthen the innovation of Changle Story in genres and styles[4]. Changle Story should use historical stories for reference, and explore the elements that the public are delighted to see and hear and that reflects the spiritual outlook of the time and has great practical significance, instead of only drawing materials from something like prosperity and delivery from disaster, an abundant harvest of all crops, and the harmony of neighboring relations. Moreover, it is of significance to cultivate and bring up talents who not only have a good understanding of Changle Story but also expert in marketing and management. Finally, the innovation in marketing channel should also be strengthened. Changle Story should find its way out of its hometown to the world and develop extensive cooperation with the markets of both tourism souvenir and art, and in this way we can create a huge space for the sustainable development of Changle Story.

Make Policies on the Industrial Development of Changle Story. The local government needs to make policies and mobilize social resources to strongly support the industrial development of Changle Story. First of all, a specialized administrative office should be established to study and make strategic policies, set short-term job objectives, and take charge of daily office work. Second, the government should set preferential policies. For example, in order to gain much support from private investors, the government should offer them the best favor in the following items, such as land expropriation, capital raising, taxing and local financial subsidies, because their investment is of great significance to the industrial development of Changle Story. What's more, the government should protect the intellectual property of Changle Story by the ways of local legislation, administration, and releasing print publications of Changle Story. Meanwhile, in order to find a broader market for Changle Story, the government should take actions to counter pirated products of Changle Story and defend the real benefits of local enterprises.

\section{Summary}

As a state-level intangible cultural heritage, the cultural and economic values of Changle Story has been increasingly recognized by the public. However, the urgent job we have to do is that: firstly, we should help Changle story remain authentic even if it is faced with threats from modern civilization and commercialization; secondly, since innovation and development is essential to the 
inheriting and preservation of intangible cultural heritages, much attention should be paid to the cultivation of younger inheritors of Changle Story because the younger generation has a broader view and a stronger spirit of pioneering, and only in this way can we strengthen the inheriting of Changle Story; thirdly, the government should develop characteristic tourism of Changle Story intangible cultural heritage, and at the same time increase the cultural soft strength and tourism attractiveness of Changle Story, which will not only promote the brand recognition of Changle Story but also increase the income of local people and create more wealth for the local society. The inheriting and preservation of intangible cultures should always be centered around people, based on economy. More importantly, we should try to turn the disadvantages of Changle Story into its advantages, and make the fullest use of its advantages[5], so that we can achieve the goal of inheriting and preserving the live culture and make it longstanding.

\section{References}

[1] S.H. Yu :The Art of Floating in the Air.(Hunan People's Publishing House, Chang Sha2012,p.1)(In Chinese).

[2] D.Yu: Introduction to art management.(Higher Education Press, Beijing2008,p.124)(In Chinese).

[3] X.Z.Wu. The Thoughts on the System Design of intangible cultural heritage. South-Central University for Nationalities' Academic Journal(Humanities and Social Sciences),(2017)No.3,p.55(In Chinese).

[4] M.X.Cheng, Q.Wang. Research on the Economic Value Development Path of Intangible Cultural Heritage. Economic Aspect,(2013)No.11,p.27(In Chinese).

[5] Z.Y.Ou Yang. SWOT Analysis of Intangible Cultural Heritage Tourism in Gansu Province. Resources and Environment of Arid Land, (2011)No.7,p.208(In Chinese). 